\title{
Magnetically Directed Two-Dimensional Crystallization of OmpF Membrane Proteins in Block Copolymers
}

\author{
Steven S. Klara ${ }^{1}$, Patrick O. Saboe ${ }^{2}$, Ian T. Sines ${ }^{2}$, Mahnoush Babaei ${ }^{3}$, Po-Lin Chiu ${ }^{4}$, Rita DeZorzi ${ }^{4,5 \%}$, \\ Kaushik Dayal ${ }^{3}$, Thomas Walz ${ }^{4,5, \&}$, Manish $\operatorname{Kumar}^{2} *$, and Meagan S. Mauter ${ }^{1,3,6, *}$. \\ ${ }^{1}$ Department of Chemical Engineering, Carnegie Mellon University, Pittsburgh, PA 15213, USA, ${ }^{2}$ Department of Chemical \\ Engineering, Pennsylvania State University, University Park, PA 16802, USA, ${ }^{3}$ Deparment of Civil \& Environmental \\ Engineering, Carnegie Mellon University; ${ }^{4}$ Department of Cell Biology, Harvard Medical School, Boston, Massachusetts, United \\ States of America; ${ }^{5}$ Howard Hughes Medical Institute, Harvard Medical School, Boston, Massachusetts, United States of \\ America; ${ }^{6}$ Department of Engineering \& Public Policy, Carnegie Mellon University. ${ }^{\%}$ Present address: Dipartimento di Scienze

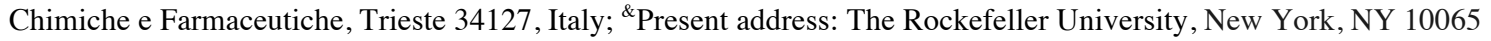

\section{AUTHOR INFORMATION}

*Corresponding Authors

Meagan Mauter: mauter@cmu.edu

Manish Kumar: manish.kumar@psu.edu 
Table of Contents

1. Chemicals

2. Experimental Procedures
a. Expression and Purification of OmpF
b. Polymer Synthesis
c. 2D Crystallization of OmpF
d. Electron Microscopy and Image Analysis

3. EM Images of OmpF 2D Crystals in BCP membranes

4. Analyses of Crystals Obtained in the Presence and Absence of a Magnetic Field

5. Calculation of Diamagnetic Anisotropy in AQP0 and OmpF

1. Chemicals: n-Octylpolyoxyethylene (Octyl-POE) was purchased from Bachem Biochemical Company. n-Octyl- $\beta$-D-glucopyranoside was purchased from Anatrace. 4-(2Hydroxyethyl)piperazin-1-ylethanesulphonic acid (HEPES), 2-(N-morpholino)ethanesulfonic acid (MES), sodium chloride, magnesium chloride, 1-naphthol, and D,L-dithiothreitol (DTT) were purchased from Amresco Biochemical Company. Sodium azide was purchased from EMD Millipore. Ethylene oxide was obtained from Balchem Corporation and 1-3 butadiene from Sigma Aldrich. Sec-butyl lithium was purchased from Sigma. Solvents for polymer synthesis were obtained from EMD Millipore.

2A. Expression and Purification of OmpF: E. coli BL21(DE3)omp $8^{l}$ cells were grown in a 60L fermentor in Luria-Bertani (LB) broth with $0.5 \%$ glycerol and $50 \mathrm{mg} / \mathrm{L}$ ampicillin using an agitation of $300 \mathrm{rpm}$. At an $\mathrm{OD}_{600}$ of 2 , cells were induced with $0.02 \mathrm{mM}$ isopropyl- $\beta$-D- 
thiogalactoside (IPTG) and the temperature was reduced to $16^{\circ} \mathrm{C}$ for protein expression. About 1 $\mathrm{kg}$ of wet cells were harvested after 18 hours of growth and stored at $-80^{\circ} \mathrm{C}$. Frozen cells $(1 \mathrm{~g}$ of cell pellet per $10 \mathrm{~mL}$ of buffer) were suspended in $20 \mathrm{mM}$ Tris, $\mathrm{pH} 8.0$, with $1 \mathrm{U} / \mu \mathrm{L}$ DNase and lysed with a microfluidizer. Unbroken cells were spun down for $15 \mathrm{mins}$ at $4000 \mathrm{~g}$. The broken cells were incubated with $1 \%$ SDS for 20 mins, and cell membranes were spun down for 60 mins at $200,000 \mathrm{~g}, 4^{\circ} \mathrm{C}$. Membranes were resuspended in $0.125 \%$ octyl-POE, $20 \mathrm{mM}$ sodium phosphate, $\mathrm{pH} 7.4$, using $5 \mathrm{~mL}$ buffer per $1 \mathrm{~g}$ of cells, and incubated for $60 \mathrm{~min}$ at $37^{\circ} \mathrm{C}$. Membranes were spun down for $60 \mathrm{~min}$ at $200,000 \mathrm{~g}, 4^{\circ} \mathrm{C}$. Extraction was completed by incubating resuspended membranes with $3 \%$ octyl-POE in $20 \mathrm{mM}$ phosphate buffer, $\mathrm{pH} 7.4$ (2 $\mathrm{mL}$ buffer per $1 \mathrm{~g}$ cell pellet) for 1 hour at $37^{\circ} \mathrm{C}$. Unsolubilized membranes were spun down for 30 mins at $200,000 \mathrm{~g}, 4^{\circ} \mathrm{C}$. The supernatant was loaded on to a HiScreen DEAE FF column (GE Healthcare, Pittsburgh, PA), washed with $5 \mathrm{mM} \mathrm{Na}_{3} \mathrm{PO}_{4}, \mathrm{pH} 7.6,3 \mathrm{mM} \mathrm{NaN} 3,1 \%$ octyl-POE and then eluted with $5 \mathrm{mM} \mathrm{Na}_{3} \mathrm{PO}_{4}, \mathrm{pH}$ 7.6, $3 \mathrm{mM} \mathrm{NaN}_{3}, 1 \%$ octyl-POE, $10 \mathrm{mM}$ EDTA. The eluted peak fractions were pooled and run over a Superose 12 column (GE Healthcare) following a procedure developed by Garavito and Rosenbusch. ${ }^{2}$ Size-exclusion fractions were concentrated, and the protein content was quantified by using the Bradford assay.

2B. Polymer Synthesis: Polyethylethylene- $b$-polyethylene oxide block copolymers were synthesized using anionic polymerization, based on a procedure described by Bates and coworkers $^{3}$ with slight modifications. First, synthesis of 1,2-polybutadiene was conducted in tetrahydrofuran using sec-butyl lithium as initiator at $-65^{\circ} \mathrm{C}$. Polymerization was terminated by addition of ethylene oxide, yielding monohydroxyl-terminated polybutadiene. Polyethylene oxide growth was accomplished by converting the hydroxyl group to potassium alkoxide, which 
was used as a macroinitiator. Polyethylethylene-b-polyethylene oxide block copolymers were then synthesized using a hydrogenation step to convert polybutadiene to polyethylethylene. Hydrogenation was performed using a high-pressure Parr reactor and a palladium catalyst. Block composition and lengths were estimated using ${ }^{1} \mathrm{H}$ NMR, and the lengths were confirmed by gel permeation chromatography. The final block composition was determined to be $\mathrm{PB}_{12^{-}}$ $\mathrm{PEO}_{8}$ with a polydispersity index of 1.18 .

2C. 2D Crystallization of OmpF: A crystallization buffer of $20 \mathrm{mM}$ HEPES, pH 7.2, $10 \mathrm{mM}$ $\mathrm{MgCl}_{2}, 100 \mathrm{mM} \mathrm{NaCl}, 0.2 \mathrm{mM}$ DTT, and $3 \mathrm{mM} \mathrm{NaN}_{3}{ }^{4}$ was used to incubate $60-\mu \mathrm{L}$ dialysis buttons (Hampton Research, Aliso Viejo, CA). Polymer-to-protein ratios (PoPRs) of 0.1-1.0 were tested. Crystallization buffer with $4 \%(\mathrm{w} / \mathrm{v})$ octyl-POE was added to the samples to bring each button to a final volume of $60 \mu \mathrm{L}$. The final protein concentration in each button was 1 $\mathrm{mg} / \mathrm{mL}$. The buttons were covered with a 12000-14000 MWCO dialysis membrane (Spectrum Labs, Irving, TX) and sealed with a rubber O-ring.

The samples were placed into $50 \mathrm{~mL}$ of buffer solution containing $4 \%$ (w/v) octyl-POE. The detergent concentration was halved every 24 hrs by adding detergent-free dialysis buffer. At an ocytl-POE concentration of $0.25 \%$, the samples were transferred into the homogeneous magnetic field from a horizontal-bore, Magnex Scientific, 7.5 Tesla (T), $21 \mathrm{~cm}$, superconducting magnet with a passive iron shield field for 24 hours at room temperature in $50 \mathrm{~mL}$ of crystallization buffer containing $0.25 \%$ octyl-POE. The buffer solution was then replaced with $50 \mathrm{~mL}$ of fresh buffer without octyl-POE, and left in the electromagnet for an additional 24 hours. After removal from the electromagnet, residual detergent was removed by dialysis against $1 \mathrm{~L}$ of dialysis buffer, which was renewed every 4 hours for 3 times. 
2D. Electron Microscopy and Image Analysis: For negative staining, crystal suspensions were adsorbed on a glow-discharged carbon-coated electron microscopy grid, negatively stained with 0.75\% (w/v) uranyl formate, and imaged with an FEI Tecnai G2 Spirit BioTwin electron microscope operated at $80 \mathrm{kV}$ and using an FEI Eagle 4K x 4K HS CCD or Gatan Orius 2K x 2K CCD camera. The CCD cameras were calibrated using AQP0 2D crystals. Image analysis of the crystals was performed using the $2 d x^{5}$ and Image ${ }^{6}$ software packages.

For cryo-EM, OmpF 2D crystals were prepared using a variation of the carbon sandwich technique $^{7}$ on molybdenum grids with $7 \%(\mathrm{w} / \mathrm{v})$ trehalose solution, and the grids were plungefrozen in liquid nitrogen. Crystals were imaged with a Polara electron microscope (FEI Company, Hillsborough, OR) operated at $300 \mathrm{kV}$, using a defocus range of -0.2 to $-0.5 \mu \mathrm{m}$. Data were collected using low-dose procedures on a K2 Summit camera (Gatan Inc., Pleasanton, CA). Images were acquired and processed as described. ${ }^{8}$ 


\section{EM Images of OmpF 2D Crystals in BCP membranes}
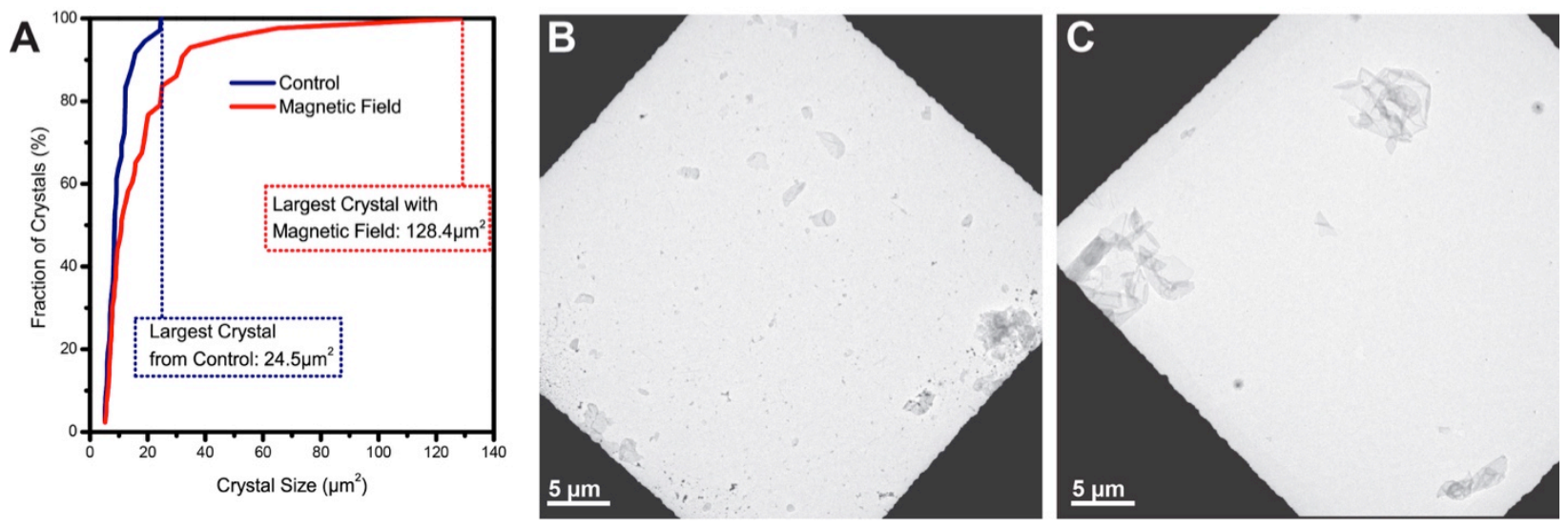

Figure S1: A) Cumulative distribution function of crystal size for crystals larger than $5 \mu \mathrm{m}^{2}$ for both control (Control) and magnetically aligned (Magnetic Field) samples. B) Representative low-magnification EM image of control crystals grown in the absence of a magnetic field. C) Representative low-magnification EM image of crystals grown in the presence of a magnetic field. 


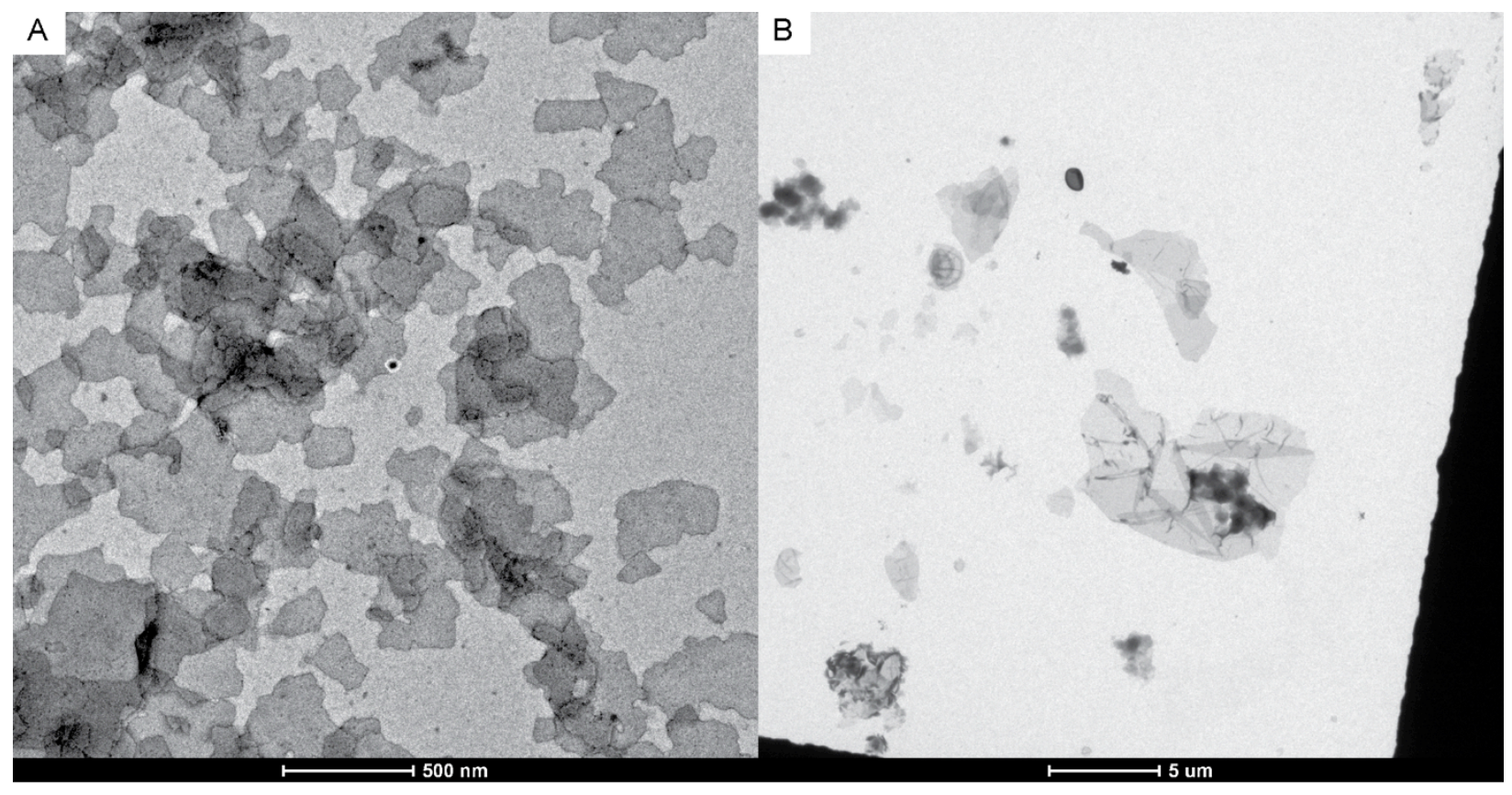

Figure S2: Representative EM images of negatively stained OmpF 2D crystals grown in the absence (A) and presence (B) of a magnetic field. Note that the scale bars are $500 \mathrm{~nm}$ in (A) and $5 \mu \mathrm{m}$ in (B). 


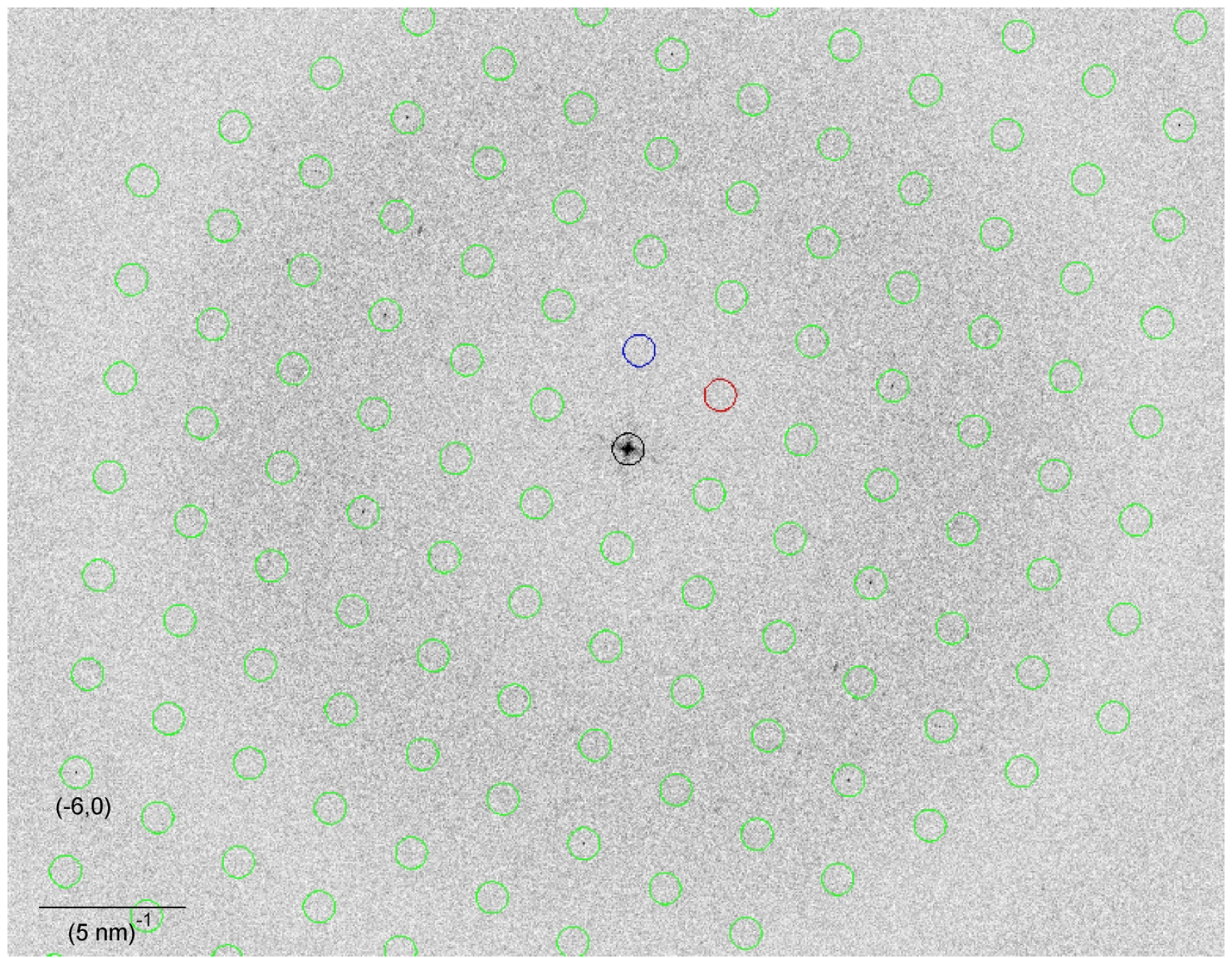

Figure S3: Fourier transform of a cryo-EM image of a sugar-embedded OmpF 2D crystal grown in the absence of a magnetic field. The Fourier transform shows 6 orders of diffraction spots. 


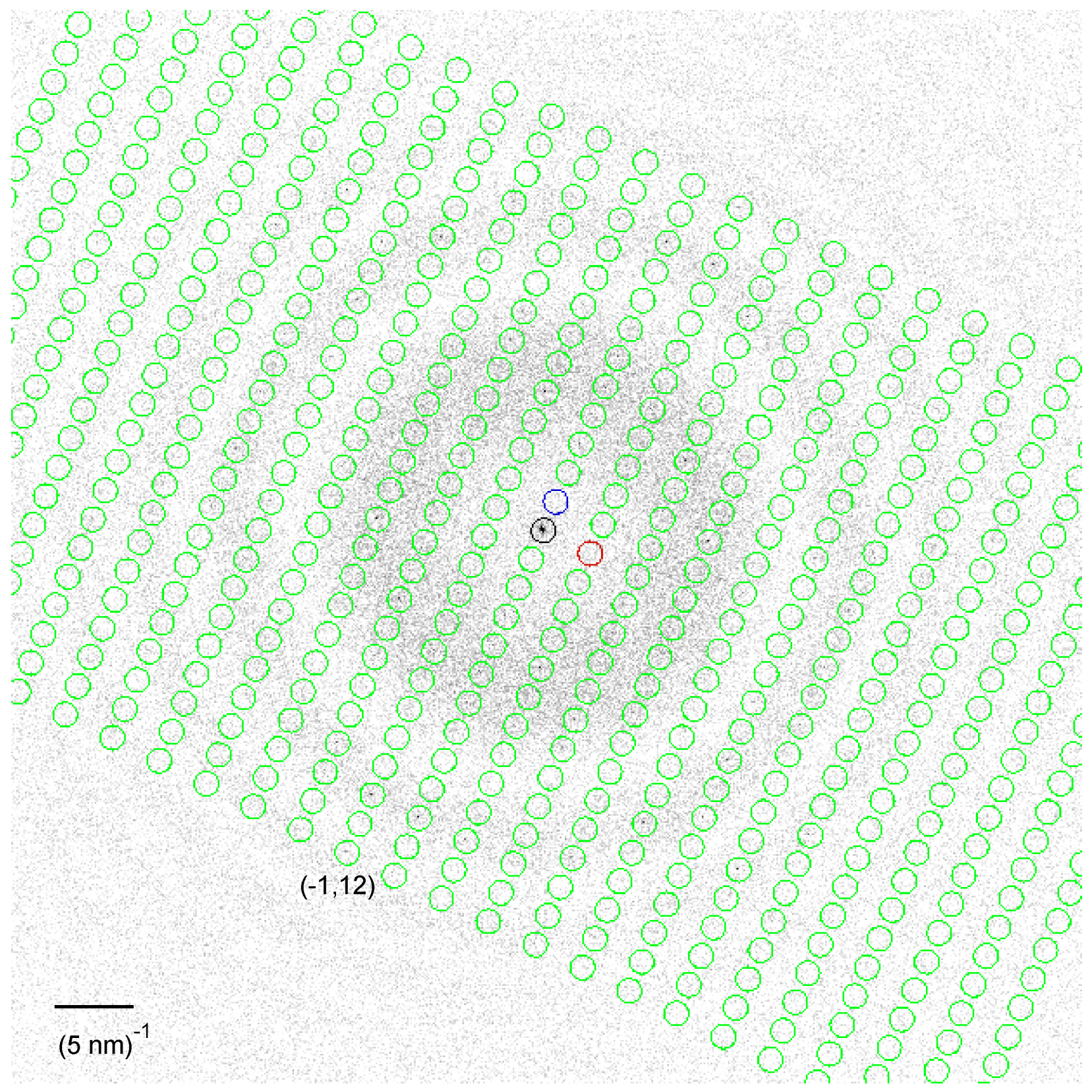

Figure S4: Fourier transform of a cryo-EM image of a sugar-embedded OmpF 2D crystal grown in the presence of a magnetic field of $7.5 \mathrm{~T}$. The Fourier transform shows 12 orders of diffraction spots. 


\section{Analyses of Crystals Obtained in the Presence and Absence of a Magnetic Field}

Table S1. Analysis of the quality of OmpF 2D crystals grown in the presence (Aligned) and absence (Unaligned) of a magnetic field based on the signal-to-noise ratio of reflections in Fourier transforms of EM images after computational unbending and background subtraction.

\begin{tabular}{cccc}
\hline \multirow{2}{*}{ IQ } & P(IQ) & \multicolumn{2}{c}{ Number of FFT spots } \\
\cline { 3 - 4 } & & Aligned & Unaligned \\
\hline $\mathbf{1}$ & - & $24.7 \pm 5.6$ & $8.6 \pm 5.8$ \\
$\mathbf{2}$ & $<0.0001$ & $23.2 \pm 9.0$ & $12.8 \pm 5.6$ \\
$\mathbf{3}$ & 0.0002 & $12.9 \pm 5.6$ & $11.8 \pm 6.4$ \\
$\mathbf{4}$ & 0.008 & $21.8 \pm 7.6$ & $20.6 \pm 7.8$ \\
$\mathbf{5}$ & 0.066 & $31.4 \pm 6.5$ & $36.6 \pm 8.2$ \\
\hline
\end{tabular}

Note: The signal-to-noise ratio of reflections in Fourier transforms (represented by the IQ values) depends on the size of the crystal areas used to calculate the Fourier transforms (see Table S2). Since crystals grown in the presence of a magnetic field are larger than the control crystals, the statistics in this table are biased in favor of the crystals grown in the presence of a magnetic field. 
Table S2. Effect of the crystal area used for image analysis on the quality and number of reflections seen in the resulting Fourier transform. For this analysis, an image of a crystal was used that was grown in the presence of a magnetic field. The crystal was computationally unbent and the background was subtracted from the Fourier transforms. Note that the number of high-SNR (low-IQ) spots increases with the size of the crystal area that was analyzed.

\begin{tabular}{cccc}
\hline & \multicolumn{3}{c}{ Number of FFT spots } \\
\cline { 2 - 4 } $\mathbf{Q}^{*}$ & $\mathbf{0 . 1 6} \boldsymbol{\mu \mathbf { m } ^ { \mathbf { 2 } }}$ & $\mathbf{0 . 3 6} \boldsymbol{\mu \mathbf { m } ^ { \mathbf { 2 } }}$ & $\mathbf{0 . 6 4} \boldsymbol{\mu \mathbf { m } ^ { \mathbf { 2 } }}$ \\
\hline $\mathbf{1}$ & 8 & 20 & 28 \\
$\mathbf{2}$ & 37 & 35 & 35 \\
$\mathbf{3}$ & 16 & 12 & 10 \\
$\mathbf{4}$ & 12 & 17 & 18 \\
$\mathbf{5}$ & 30 & 36 & 33 \\
\hline
\end{tabular}

Table S3. Additional statistics on the number and distribution of reflections presented in Table 1. Ten crystals were analyzed for both aligned and unaligned samples, and errors are represented by standard deviation.

\begin{tabular}{ccccc}
\hline & \multicolumn{4}{c}{ Number of reflections } \\
\cline { 2 - 5 } $\mathbf{I Q}^{*}$ & \multicolumn{2}{c}{ Aligned } & \multicolumn{2}{c}{ Unaligned } \\
\cline { 2 - 5 } & $>\mathbf{2 . 4 \AA}$ & $\mathbf{2 . 4 \AA}$ & $\mathbf{2 . 4 \AA}$ & $\leq \mathbf{2 . 4 \AA}$ \\
\hline $\mathbf{1}$ & $5.6 \pm 1.5$ & 0.0 & $2.1 \pm 2.6$ & 0.0 \\
$\mathbf{2}$ & $9.2 \pm 3.5$ & $0.2 \pm 0.4$ & $6.4 \pm 6.5$ & $0.2 \pm 0.6$ \\
$\mathbf{3}$ & $7.0 \pm 4.1$ & $0.7 \pm 0.7$ & $5.1 \pm 4.4$ & $0.5 \pm 0.7$ \\
$\mathbf{4}$ & $7.9 \pm 2.9$ & $4.6 \pm 6.8$ & $5.4 \pm 3.2$ & $1.6 \pm 1.4$ \\
$\mathbf{5}$ & $6.0 \pm 2.0$ & $8.1 \pm 11.4$ & $5.8 \pm 2.9$ & $1.6 \pm 1.4$ \\
\hline
\end{tabular}




\section{Calculation of Diamagnetic Anisotropy in AQP0 and OmpF}

Table S4: Critical volume, area, diameter, and number of $\mathrm{AQP0}$ and OmpF proteins, for which magnetic stabilization energy exceeds $k T$.

\begin{tabular}{|c|c|c|c|c|}
\hline Protein Type & AQP0 & AQP0 Tetramer & $\mathrm{OmpF}$ & OmpF Trimer \\
\hline \multicolumn{5}{|l|}{ Schematic View } \\
\hline Yolume $\left(4^{3}\right)$ & 3020223 & 12080807 & 4241570 & 12721737 \\
\hline Structure Weight $(\mathrm{g} / \mathrm{mol})$ & 25173.60 & 100694.40 & 4079191 & 12237570 \\
\hline Protein molar DA, $\Delta \chi_{m}($ emu-cgs) & $-4.85 \mathrm{E}-04$ & $-1.26 \mathrm{E}-03$ & $-1.40 \mathrm{E}-03$ & $-3.35 \mathrm{E}-03$ \\
\hline $\begin{array}{c}\text { Protein volumetric DA, } \Delta \chi \\
\text { (dimensionless) }\end{array}$ & $3.35 \mathrm{E}-07$ & $2.18 \mathrm{E}-07$ & $6.89 \mathrm{E}-07$ & $5.49 \mathrm{E}-07$ \\
\hline Volume $_{\min }$ For B $=7.5 \mathrm{~T}\left(\mathrm{~nm}^{3}\right)$ & $5.36 \mathrm{E}+05$ & $8.25 \mathrm{E}+05$ & $2.61 \mathrm{E}+05$ & $3.27 \mathrm{E}+05$ \\
\hline Volume $_{\min }$ For $\mathrm{B}=15 \mathrm{~T}\left(\mathrm{~nm}^{3}\right)$ & $1.34 \mathrm{E}+05$ & $2.06 \mathrm{E}+05$ & $6.52 \mathrm{E}+04$ & $8.14 \mathrm{E}+04$ \\
\hline Area $_{\min }$ For B $=7.5 \mathrm{~T}\left(\mathrm{~nm}^{2}\right)$ & $9.87 \mathrm{E}+04$ & $1.52 \mathrm{E}+05$ & $4.48 \mathrm{E}+04$ & $5.62 \mathrm{E}+04$ \\
\hline Area $_{\min }$ For $B=15 \mathrm{~T}\left(\mathrm{~nm}^{2}\right)$ & $2.47 \mathrm{E}+04$ & $3.80 \mathrm{E}+04$ & $1.12 \mathrm{E}+04$ & $1.40 \mathrm{E}+04$ \\
\hline Diameter $_{\min }$ For $\mathrm{B}=7.5 \mathrm{~T}(\mathrm{~nm})$ & 314.1796 & 389.8464 & 211.6740 & 237.0120 \\
\hline Diameter $_{\min }$ For $\mathrm{B}=15 \mathrm{~T}(\mathrm{~nm})$ & 157.0898 & 194.9232 & 105.8370 & 118.5060 \\
\hline Number $_{\min }$ For $\mathrm{B}=7.5 \mathrm{~T}$ & 17748 & 6832 & 6148 & 2570 \\
\hline Number $_{\min }$ For $\mathrm{B}=15 \mathrm{~T}$ & 4437 & 1708 & 1537 & 643 \\
\hline
\end{tabular}




\section{References}

(1) Prilipov, A.; Phale, P. S.; Gelder, P.; Rosenbusch, J. P.; Koebnik, R. FEMS Microbiol. Let. 1998, 163, 6572.

(2) Garavito, R. M.; Rosenbusch, J. P. Method. Enzymol. 1986, 125, 309-328.

(3) Hillmyer, M. A.; Bates, F. S. Macromolecules 1996, 29, 6994-7002.

(4) Hoenger, A.; Gross, H.; Aebi, U.; Engel, A. J. Struct. Biol. 1990, 103, 185-195.

(5) Gipson, B.; Zeng, X.; Zhang, Z. Y.; Stahlberg, H. J. Struct. Biol. 2007, 157, 64-72.

(6) Abràmoff, M. D.; Magalhães, P. J.; Ram, S. J. Biophotonics International 2004, 11, 36-42.

(7) Gyobu, N.; Tani, K.; Hiroaki, Y.; Kamegawa, A.; Mitsuoka, K.; Fujiyoshi, Y. J. Struct. Biol. 2004, 146, 325-333.

(8) Chiu, P.-L.; Li, X.; Li, Z.; Beckett, B.; Brilot, A. F.; Grigorieff, N.; Agard, D. A.; Cheng, Y.; Walz, T. J. Struct. Biol. 2015, 192, 163-173 